\title{
Orientation and Motion of Water Molecules at Air/Water Interface
}

\author{
Wei Gan* Dan Wu* Zhen Zhang * and Hong-fei Wangt \\ State Key Laboratory of Molecular Reaction Dynamics, \\ Institute of Chemistry, the Chinese Academy of Sciences, Beijing, China, 100080
}

(Dated: November 9, 2018)

\begin{abstract}
Analysis of SFG vibrational spectra of $\mathrm{OH}$ stretching bands in four experimental configurations shows that orientational motion of water molecule at air/water interface is libratory within a limited angular range. This picture is significantly different from the previous conclusion that the interfacial water molecule orientation varies over a broad range within the vibrational relaxation time, the only direct experimental evidence for ultrafast and broad orientational motion of a liquid interface by Wei et al. [Phys. Rev. Lett. 86, 4799, (2001)] using single SFG experimental configuration.
\end{abstract}

PACS numbers: $33.20 . \mathrm{Tp}, 42.65 . \mathrm{An}, 68.35 . \mathrm{Ja}, 82.30 . \mathrm{Rs}$

Due to lack of direct experimental measurement techniques, motion and dynamics of molecules at liquid interface are yet to be well understood [1]. In the past decade, air/water interface, as one of the most important liquid interface, has been intensively investigated both theoretically and experimentally $1,2,3,4,5,6$, 7, 8, 9, 10, 11, 12, 13, 14]. Experimental method such as X-Ray reflection can be used to get the liquid surface roughness [2], but orientational structure and dynamics information at molecule level can only be obtained by Sum Frequency Generation (SFG) or Second Harmonic Generation (SHG) because of their submonolayer sensitivity and interface specificity [1]. It has been generally accepted that the interfacial water molecule has one free $\mathrm{OH}$ bond protruding out of the interface. However, the existence of water molecules with both $\mathrm{OH}$ bonds pointing to the vapor phase is still a controversial issue 5, 8, 9, 10, 14. The dynamics of water molecule at air/water interface has been discussed theoretically in literatures [4, 6, 7, 11, 13, 14]. The only direct experimental study so far concluded a fast orientational motion varies over a broad range of $102^{\circ}$ centered at the surface normal $\left(\theta_{M}=51^{\circ}\right)$ within a time scale comparable or less than $0.5 \mathrm{ps}$, by Wei et al. from the fact of the vanishing of the SFG vibrational spectra of the free $\mathrm{OH}$ stretching mode of the interfacial water molecule around $\sim 3700 \mathrm{~cm}^{-1}$ in the sps polarization (denoting $s$ - $p$-, and $s$ - polarized sum frequency output, visible input, and infrared input, respectively) [9]. This result indicated a very dynamic and disordered physical picture for the air/water interface.

Femtosecond pump-probe experimental studies have shown that orientational relaxation dynamics of water molecules in bulk water is as fast as 1ps, and also the motion of non-hydrogen bonded water molecules is faster than hydrogen bonded water molecules [15]. Therefore, it is reasonable to predict very fast orientational motion of interfacial water molecules, which is less hydrogen bonded as bulk water molecules. However, theoretical simulation by Chandra et al. argued for slower dynamics for interfacial water molecules than that of bulk ones (10.5ps vs. 7.1ps) 13]. Simulation by Benjamin et al. also suggested that the lifetime of hydrogen bond at interface between water and an organic liquid is significantly longer than that of bulk water molecules 16 . No matter how, these all suggested ultrafast orientational motion of interfacial water molecules. Most importantly, ultrafast orientational motion may have effects on vibrational spectral width, as well as the strength of vibrational resonance in different input/output polarization combinations, provided that the motion is faster than the time scale of $1 / \Gamma_{q}$ (the rapid motion limit) and the motion covers a broad range centered around the surface normal, as reported by Wei et al. [9].

Recent quantitative analysis of data in SFG vibrational spectroscopy have suggested that vapor/liquid interface are generally well ordered, and sometimes even with antiparallel double-layered structures 1, 17, 18, 19]. It has been generally accepted that liquid interface with strong hydrogen bonding between molecules should be well ordered [1]. Therefore, it is surprising to comprehend the picture dictated by ultrafast orientational motion in such a broad angular range.

Here we show from SFG-VS measurements in four sets of experimental configurations that Wei et al.'s analysis [9] can only be considered valid for the particular SFG experimental configuration. We conclude that the orientational motion of water molecules at the air/water interface can only be libratory within a limited angular range around a tilting angle around $33^{\circ}$, instead of being around the interface normal. Therefore, the air/water interface is much better ordered than previously suggested.

The detail of the SFG-VS experiment was described elsewhere [20]. Fig. 1]shows SFG spectra of the air/water interface in reflective geometry with $s s p, p p p$ and $s p s$ polarization combinations at four sets of incident angles for the visible(Vis) and IR laser beams, namely, Config.1: $\mathrm{Vis}=39^{\circ}, \mathrm{IR}=55^{\circ}$; Config. $2: \mathrm{Vis}=45^{\circ}, \mathrm{IR}=55^{\circ}$; Config. 3: Vis $=48^{\circ}, \mathrm{IR}=57^{\circ}$; Config. 4 : $\mathrm{Vis}=63^{\circ}, \mathrm{IR}=55^{\circ}$. It is clear that there is strong dependence of $p p p$ and sps spectra on the incident angles, especially on the visible 
beam. The sps spectra in Config. 4 shows clearly much larger intensity at $\sim 3700 \mathrm{~cm}^{-1}$ than those of the other three configurations. Even though the sps intensity is generally much smaller than that of $s s p$ and $p p p$ polarizations, the sps intensity is well above the experimental error, and have been repeated more than a dozen times. It is important to see that the spectra in Config. 2 resembles closely the data obtained by Wei et al. with $\mathrm{Vis}=45^{\circ}, \mathrm{IR}=57^{\circ}[$ [] $]$.

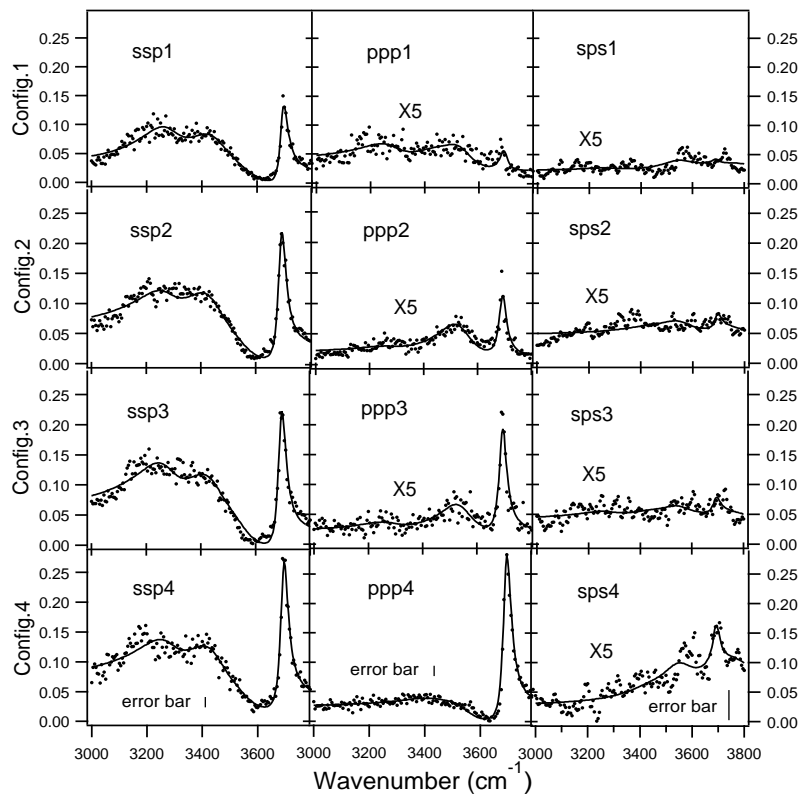

FIG. 1: SFG spectra of air/water interface in different polarization combination and experimental configurations. All spectra are normalized to the same scale. The solid lines are globally fitted curves with Lorentzian line shape function. Note the different error bars for graphs in different scales.

The ssp spectra of all four experimental configurations overlap with each other well within experimental error when normalized to the $\sim 3700 \mathrm{~cm}^{-1}$ peak intensity. The difference of their absolute intensities can be quantitatively accounted from the Fresnel coefficients with different incident angles. These facts indicate that SFG data with different incident angles are from interfacial layers without detectable bulk contributions 21].

Quantitative polarization and orientational analysis in different experimental configurations can provide detailed information on structure, conformation and dynamics of molecules at liquid interfaces $9,22,23,24,25]$. The polarization dependent SFG intensity is [22]:

$$
I(\omega)=\frac{8 \pi^{3} \omega^{2} \sec ^{2} \beta}{c^{3} n_{1}(\omega) n_{1}\left(\omega_{1}\right) n_{1}\left(\omega_{2}\right)}\left|\chi_{e f f}^{(2)}\right|^{2} I\left(\omega_{1}\right) I\left(\omega_{2}\right)
$$

with $\chi_{e f f}^{(2)}=[\hat{e}(\omega) \cdot \mathbf{L}(\omega)] \cdot \chi_{i j k}^{(2)}:\left[\mathbf{L}\left(\omega_{1}\right) \cdot \hat{e}\left(\omega_{1}\right)\right]\left[\mathbf{L}\left(\omega_{2}\right) \cdot \hat{e}\left(\omega_{2}\right)\right]$

in which $n_{i}\left(\omega_{i}\right)$ is the refractive index of phase $i$ at frequency $\omega_{i} ; \beta$ is the angle of the laser beam; $I$ is the intensity of the laser beam or the SFG signal. $\chi_{\text {eff }}^{(2)}$ is the effective second order susceptibility; $\chi_{i j k}^{(2)}$ is the macroscopic susceptibility tensor elements, determined by the macroscopic and molecular symmetry 24]; L $\left(\omega_{i}\right)$ the local field factor tensor, and $\hat{e}\left(\omega_{i}\right)$ the unit vector of the corresponding optical field, which is responsible for the experimental configuration dependence.

As Wei et al. had demonstrated 9], when orientational motion average is considered, if the orientational motion is faster than the vibrational relaxation time scale $1 / \Gamma_{q}$ of the $q$ th mode, slow motion average of the orientation motion is no longer valid. The slow motion limit gives,

$$
\chi_{i j k}^{(2)}=N_{s} \sum_{q} \sum_{\lambda \mu \nu} \frac{a_{q, \lambda \mu \nu}}{\omega_{2}-\omega_{q}+i \Gamma_{q}}\left\langle D_{i \lambda} D_{j \mu} D_{k \nu}\right\rangle
$$

and the fast motion gives,

$$
\chi_{i j k}^{(2)}=N_{s} \sum_{q} \sum_{\lambda \mu \nu} \frac{a_{q, \lambda \mu \nu}}{\omega_{2}-\omega_{q}+i \Gamma_{q}}\left\langle D_{i \lambda} D_{j \mu}\right\rangle\left\langle D_{k \nu}\right\rangle
$$

in which $N_{s}$ is the surface density of molecules; $a_{q, \lambda \mu \nu}$ and $\omega_{q}$ and $\Gamma_{q}$ are the amplitude, resonant frequency and damping constant of the $q$ th molecular vibrational mode, respectively; and $D_{l \xi}(t)=\hat{l} \cdot \hat{\xi}(t)$ is the time-dependent direction cosine matrix with $l=i, j, k$ for laboratory coordinates and $\xi=\lambda, \mu, \nu$ for molecular coordinates.

Wei et al. used the step orientational distribution function in Eq. 5] as well as other distribution functions, such as Gaussian, centered at the surface normal, and concluded for a fast motion of the interfacial free $\mathrm{OH}$ bond in a range of $\theta_{M}=51^{\circ}$ from the data obtained with Vis $=45^{\circ}, \mathrm{IR}=57^{\circ}[9]$. Otherwise, the $p p p$ and sps intensities should be comparable, given the $s s p$ intensity is about 10 times of that for $p p p$ in their SFG data.

$$
\begin{array}{lll}
f(\theta)=\text { cost } & \text { for } & 0 \leq \theta \leq \theta_{M} \\
f(\theta)=0 & \text { for } & \theta \geq \theta_{M}
\end{array}
$$

It is clear that such treatment can not explain the much stronger sps intensity around $\sim 3700 \mathrm{~cm}^{-1}$ in Config.4, even though it can seemingly explain data in Config.1,2, and 3 . Using the same parameters and calculation procedures as Wei et al, we simulated the ssp, ppp, and sps intensities with both slow motion and fast motion limit. Fig. 2 shows the results for Config.2 and Config.4. Our calculation of Config. 2 gives the same results as that by 


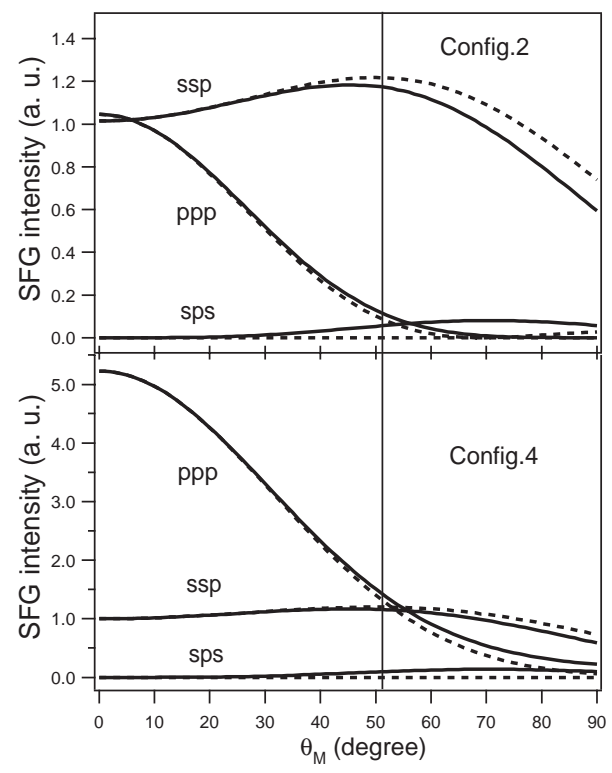

FIG. 2: SFG intensity of the free $\mathrm{OH}$ bond simulated with both slow motion limit (solid curves) and fast motion limit (dotted curves) following the procedure and parameters as Wei et al. 9]. In order to compare values in different configuration, the factor of $\sec ^{2} \beta$ is included in the calculation, and all intensities are normalized to the $s s p$ intensity in Config. 4 when $\theta_{M}=0^{\circ}$. The vertical line indicates the distribution width suggested by Wei et al.

Wei et al. [9]. We noticed in the calculation that the fast orientational motion centered at interfacial normal shall make all SFG intensity in sps spectra vanish for all experimental configurations, independent of the range of the motion. This is clearly in contradiction to the data from Config.4. As Wei et al. concluded, the slow motion limit certainly can not explain the relative intensity of the $3700 \mathrm{~cm}^{-1}$ peak for the $s s p, p p p$ polarizations. Therefore, alternative description of the motion and orientation has to be invoked.

Now we consider the case when $f(\theta)$ is a Gaussian function around $\theta_{0}$ instead of the interfacial normal.

$$
f(\theta)=\frac{1}{\sqrt{2 \pi \sigma^{2}}} e^{-\left(\theta-\theta_{0}\right)^{2} / 2 \sigma^{2}}
$$

Fig. 3 shows simulation of Config.2 and Config.4 assuming a rotationally isotropic interface with $\sigma=0$, using the same set of parameters as Wei et al. [9], and the average procedures [9, 18, 24, 26]. For data in all four configurations in Fig. 1] a consistent $\theta_{0}=33^{\circ}$ can be reached from polarization and orientation analysis [20]. This result is in good agreement with the $<38^{\circ}$ tilt angle reported by Q. Du et al. [5].

It is easy to show that when $\sigma=0$, fast motion and slow motion treatment would converge. Further simulations show that in order to satisfy the intensities of around $3700 \mathrm{~cm}^{-1}$ in all four sets of data, $\sigma$ can not exceed $15^{\circ}$ and $\theta_{0}$ changes from $33^{\circ}$ to $30^{\circ}$ as $\sigma$ increases. Within such a relatively small range of $\sigma$, simulation results for fast and slow motions become indistinguishable. Using step function of $f(\theta)$ centered at $\theta_{0}$ does not change our general conclusion. This clearly indicates that the assumption of $f(\theta)$ centered at interfacial normal is out of the question.

Libration dynamics of the hydrogen bond in liquid water can be as fast as $0.1 \mathrm{ps}[12,27$. Therefore, from the observed SFG data our general conclusion is that such extremely fast libration dynamics of the tilted free $\mathrm{OH}$ bond at the air/water interface is only possible for orientational motion within a relatively narrow range.

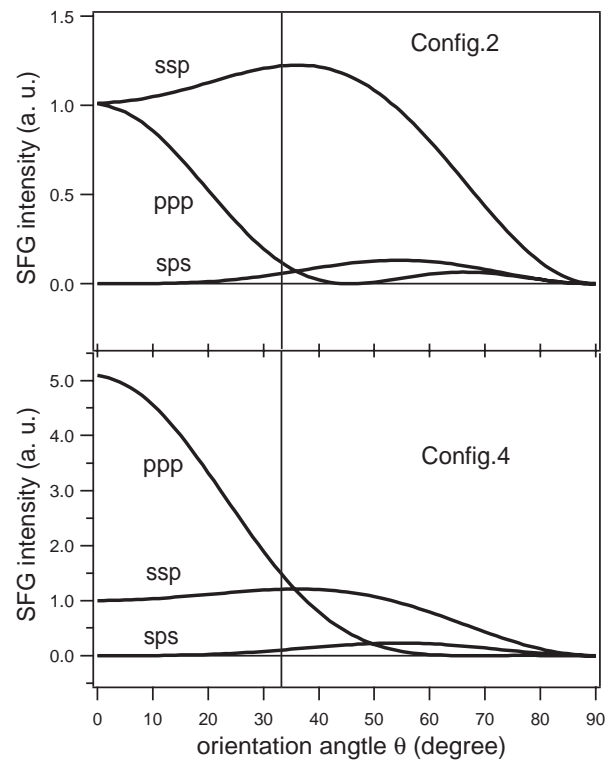

FIG. 3: Simulated SFG intensity of the free $\mathrm{OH}$ bonds at different tilt angle $\theta_{0}$ with $\sigma=0^{\circ}$. All curves are normalized to the $s s p$ intensity in Config. 4 with $\theta=0^{\circ}$. The vertical line indicates the orientation which quantitatively explains the observed SFG data. Please note the difference of the scales for the two configurations.

In the SFG literatures on air/water interface, data were usually presented for the $s s p$ polarization, except for very few cases [9, 19]. Polarization analysis were only performed by Wei et al. Our simulation shows strong dependence for the $p p p$ intensity of the free $\mathrm{OH}$ bond spectra on the visible incident angle, especially when around $\mathrm{Vis}=45^{\circ}$. In this range, one or two degree change of the visible incident angle can cause significant percentage change of the $p p p$ intensity, as can be indicated from data in Config 2 and 3. Wei et al.'s SFG experiment was performed with Vis $=45^{\circ}$. It happened to be that their analysis with ultrafast motion in a broad range of orientation centered around interface normal could just well explain this set of data. Therefore, they did not consider other possible physical pictures [28]. Besides, Richmond 
et al. suggested two possible qualitative explanations for the lack of sps intensity of the free $\mathrm{OH}$ stretching mode in the SFG measurement [29].

Polarization analysis can further determine the symmetry property of SFG spectral features [24]. Analysis indicates that both the sharp peak at $\sim 3700 \mathrm{~cm}^{-1}$ and the broad peak at $\sim 3550 \mathrm{~cm}^{-1}$ belong to $C_{\infty v}$ symmetry; while both the hydrogen bonded broad peak $\sim 3250 \mathrm{~cm}^{-1}$ and $\sim 3450 \mathrm{~cm}^{-1}$ belong to symmetric type of the $C_{2 v}$ symmetry. Analysis also indicates that the existence of interfacial water molecules with two free $\mathrm{OH}$ bonds is not supported in SFG data 20]. Such interfacial water molecule shall have $C_{2 v}$ symmetry, which should have shown clear ssp spectral intensity around $3650 \mathrm{~cm}^{-1}$ or much stronger $p p p$ spectral intensity at the asymmetric stretching mode position of $3750 \mathrm{~cm}^{-1}$ in at least one of the four sets of SFG data [8, 14].

Thus, $\sim 3550 \mathrm{~cm}^{-1}$ peak with $C_{\infty v}$ symmetry, which is apparent in the $p p p$ polarization of Config.2 and Config.3, can be assigned to the hydrogen bonded $\mathrm{OH}$ of the interfacial water molecule with a free $\mathrm{OH}$ bond. This is also supported with the fact that $3550 \mathrm{~cm}^{-1}$ is also the frequency of the hydrogen bonded $\mathrm{OH}$ in water dimer [30]. Orientational analysis of its peak intensities in different experimental configurations indicates that this $\mathrm{OH}$ bond oriented with an angle around $\sim 140^{\circ}$ from the interface normal. Indeed, with the free $\mathrm{OH}$ pointing to the vapor phase around $35^{\circ}$, the hydrogen bonded $\mathrm{OH}$ of the same molecule pointing into the liquid phase has to assume an orientation around $\sim 140^{\circ}$. This is consistent with the conclusion that the dipole vector of the interfacial water molecule is close to parallel to the interface [4, 7, 14, 31]. The detail of this analysis shall be presented elsewhere.

In summary, quantitative analysis of the SFG spectra in different polarization and experimental configurations for the air/water interface shows that orientational motion of the interfacial water molecule is libratory, as fast as 0.1 ps it might be [12, 27], only within a limited angular range of less than $15^{\circ}$ with the tilt angle around $30^{\circ}$. Therefore, the air/water interface is quite well-ordered. This picture is significantly different from the previous conclusion that the interfacial water molecule orientation varies over a broad range within the vibrational relaxation time, by Wei et al. using single SFG experimental configuration 9]. This progress provided a direct and detailed physical picture of the orientation and motion for the air/water interface.

This work was supported by Chines Academy of Sciences (No. CMS-CX200305), National Natural Science Foundation of China (NSFC No.20425309) and Chinese Ministry of Science and Technology (MOST No. G1999075305). H.F.W. acknowledges Y. R. Shen for helpful discussions.
* Also Graduate School of the Chinese Academy of Sciences

$\dagger$ Electronic address: hongfei@mrdlab.icas.ac.cn Author to whom correspondence should be addressed.

[1] P. B. Miranda, and Y. R. Shen, J. Phys. Chem. B 103, 3292-3307 (1999).

[2] A. Braslau et al., (a) Phys. Rev. Lett. 54, 114-117 (1985); (b) Phys. Rev. A 38, 2457-2470 (1988).

[3] M. C. Goh et al., J . Phys. Chem. 92, 5074-5075 (1988).

[4] R. M. Townsend, and S. A. Rice, J. Chem. Phys. 94, 2207-2218 (1991).

[5] Q. Du, R. Superfine, E. Freysz, and Y. R. Shen, Phys. Rev. Lett. 70, 2313-2316 (1993).

[6] I. Benjamin, (a) Phys. Rev. Lett. 73, 2083-2086 (1994); (b) Chem. Rev. 96, 1449-1475 (1996).

[7] (a) A. Morita, and J. T. Hynes, Chem. Phys. 258, 371390 (2000); (b) J. T. Hynes, J. Phys. Chem. B 106, 673685 (2002).

[8] M. G. Brown et al., J. Phys. Chem. A 104, 10220-10226 (2000).

[9] X. Wei, and Y. R. Shen. Phys. Rev. Lett. 86, 4799-4802 (2001).

[10] K. R. Wilson et al., J. Phys.: Condens. Matter 14, L221226 (2002).

[11] A. Perry et al., J. Chem. Phys. 118, 8411-8419 (2003).

[12] C. J. Fecko et al., Science 301, 1698-1702 (2003).

[13] S. Paul, and A. Chandra, Chem. Phys. Lett. (a) 373, 87-93 (2003); (b) 386, 218-224 (2004).

[14] I-F. W. Kuo, and C. J. Mundy, Science 303, 658-660 (2004).

[15] S. Woutersen, U. Emmerichs, H. J. Bakker, Science 278, 658-660 (1997).

[16] I. Benjamin, J. Phys. Chem. B 109, (2005), jp044157f. In Press.

[17] Y. L. Yeh et al., J. Chem. Phys. 114, 1837 (2001).

[18] H. Chen et al., (a) J. Phys. Chem. B 109, 8053-8063 (2005); (b) J. Phys. Chem. B 109, 8064-8075 (2005); (c) Chem. Phys. Letts, 408, 284-289 (2005).

[19] E. Tyrode et al., J. Phys. Chem. B 109, 329-341 (2005).

[20] Detailed SFG polarization and orientation analysis on air/water interface. To be submitted to J. Chem. Phys.

[21] Y. R. Shen, Appl. Phys. B 68, 295-300 (1999).

[22] X. Zhuang et al., Phys. Rev. B 59, 12632-12640 (1999).

[23] Y. Rao, Y. S. Tao, and H. F. Wang, J. Chem. Phys. 119, 5226-5236 (2003).

[24] (a) R. Lu et al., J. Phys. Chem. B 108, 7297-7306 (2004); (b) R. Lu et al., J. Phys. Chem. B, 109, 14118-14129 (2005); (c) H. F. Wang et al., Int. Rev. Phys. Chem. (2005). In press.

[25] H. F. Wang, Chin. J. Chem. Phys. 17, 362-368 (2004).

[26] W. Gan et al., Chem. Phys. Lett. 406, 467-473 (2005).

[27] A. Luzar, and D. Chandler, Phys. Rev. Lett. 76, 928-931 (1996).

[28] The dangling $\mathrm{OH}$ bond at the (0001) face of the hexagonal ice $\left(I_{h}\right)$ points straight up along the interface normal [Phys. Rev. Lett., 2001, 86, 1554]. Since air/water interface SFG spectra has ice-like features as in SFG of the ice surface, it was natural to assume that the free $\mathrm{OH}$ bond orientation is centered along the interface normal with a broad distribution. Private discussion with Professor Y. R. Shen. 
[29] E. A. Raymond et al., J. Phys. Chem. B 107, 546-556 (2003).

[30] R. H. Page et al., Chem. Phys. Lett. 106, 373-376 (1984).
[31] E. C. Brown et al., J. Phys. Chem. B 109, 7934-7940 (2005). 